\title{
Advances in cutaneous vasculitis research and clinical care
}

\author{
Avrom Caplan ${ }^{1}$, Robert G. Micheletti ${ }^{2}$ \\ ${ }^{1}$ Ronald O. Perelman Department of Dermatology; New York University Grossman School of Medicine, New York, NY, USA; ${ }^{2}$ Departments of \\ Dermatology and Medicine, Perelman School of Medicine, University of Pennsylvania, Philadelphia, PA, USA \\ Contributions: (I) Conception and design: All authors; (II) Administrative support: None; (III) Provision of study materials or patients: None; (IV) \\ Collection and assembly of data: None; (V) Data analysis and interpretation: None; (VI) Manuscript writing: All authors; (VII) Final approval of \\ manuscript: All authors. \\ Correspondence to: Robert G. Micheletti, MD. 3400 Civic Center Blvd, Perelman Center for Advanced Medicine, 7 South, Room 724, Philadelphia, \\ PA 19104, USA. Email: robert.micheletti@uphs.upenn.edu.
}

\begin{abstract}
Vasculitis is characterized by inflammation and destruction of blood vessels, resulting in downstream ischemic tissue damage. Diagnosis of vasculitis is a careful exercise in clinical-pathologic correlation, depending upon the clinical manifestations, organs involved, the size of affected blood vessels, imaging, and laboratory findings. While some vasculitis subtypes may be confined to the skin, serious internal organ involvement or underlying disease states may also occur. Accordingly, the skin plays an important role in the diagnostic process and may be prognostically important in some cases, signifying more severe systemic disease. The skin also provides opportunities for tissue-based translational research, improving understanding of disease pathophysiology. Dermatologists, therefore, play a critical role in evaluating vasculitis and helping to advance vasculitis clinical care and research. Recent updates in vasculitis nomenclature and terminology, evidence-based diagnosis, pathogenesis, and investigations of targeted therapies are changing vasculitis research and leading to fundamental shifts in disease management. Treatment advances favoring evidencebased and targeted, rather than broadly immunosuppressive, therapies are in development, while a multicenter trial for skin-limited vasculitis is ongoing. Collaborative multidisciplinary research networks are key to current and future advances in vasculitis research. In this review, we describe recent developments in vasculitis clinical care and research, starting with a discussion of efforts to develop diagnostic and classification criteria, followed by updates on the evaluation and treatment of vasculitis.
\end{abstract}

Keywords: Vasculitis; cutaneous vasculitis; nomenclature; targeted therapy; NETosis

Submitted Sep 13, 2020. Accepted for publication Feb 03, 2021.

doi: $10.21037 / \mathrm{atm}-20-6395$

View this article at: http://dx.doi.org/10.21037/atm-20-6395

\section{Introduction}

Vasculitis is characterized by inflammation and destruction of blood vessels, leading to ischemic tissue damage. Unlike vasculopathy, which results from clotting and mechanical occlusion of blood vessels, vasculitis is marked by inflammatory destruction. The cutaneous manifestations of vasculitis and vasculopathy may overlap or appear similar, but their systemic manifestations, diseases associations, diagnostic evaluation, management, and prognosis diverge. Because the skin is one of the most frequently affected organs, dermatologists play a key role in accurate diagnosis and effective management of vasculitis and are wellpositioned to help advance clinical care and research. This manuscript provides an update on recent developments in vasculitis, with a focus on nomenclature and classification, treatment advances and targeted therapies, research priorities, and future directions.

\section{Nomenclature and classification-the Chapel Hill Consensus Conference}

The 2012 International Chapel Hill Consensus Conference (CHCC2012) provided an update and modification to the 
1994 Chapel Hill Consensus Conference nomenclature (1). CHCC2012 does not establish diagnostic criteria but provides standardized nomenclature and disease definitions based on clinical features, separating forms of vasculitis into categories based on those definitions.

This categorization is based primarily on affected blood vessels (e.g., large vessel vasculitis, medium vessel vasculitis, and small vessel vasculitis). This terminology refers to the type (structure and function), and not solely the size, of blood vessels. New to CHCC2012 is the definition of small vessel vasculitis. This category includes ANCA-associated vasculitides (AAV) and cryoglobulinemic vasculitis. Additionally, single organ vasculitis (SOV) was introduced to describe vasculitis limited to a single organ, such as skin-limited cutaneous vasculitis (cutaneous small vessel vasculitis). Patients with SOV who subsequently develop a systemic vasculitis should be recategorized.

This widely used classification system has advanced vasculitis research and care, but limitations remain.

\section{Cutaneous vasculitis-beyond Chapel Hill}

Although cutaneous vasculitis was included in CHCC2012, the specific features and varied morphologies of the different subtypes of cutaneous vasculitis were not fully addressed. A consensus group of vasculitis experts, including dermatologists, added a Dermatology Addendum to CHCC2012 (D-CHCC) to provide a nomenclature of vasculitis affecting the skin (2). This addendum reframes cutaneous vasculitis as falling within one of three categories: (I) a cutaneous component of systemic vasculitis; (II) a skinlimited or skin-predominant variant of a systemic vasculitis; or (III) a SOV of the skin that is distinct from recognized systemic vasculitides (2).

This addendum complements the CHCC2012 classification of vasculitis based on vessel size, including small vessels located in the dermis and observations regarding the morphology of skin lesions. It further emphasizes that leukocytoclastic vasculitis is a histopathologic descriptor and not a specific disease entity, and it standardizes names and definitions of skin-predominant vasculitides. These include cutaneous polyarteritis nodosa (PAN), nodular vasculitis, IgM/IgG cutaneous vasculitis, skin-limited IgA vasculitis, skin-limited cryoglobulinemic vasculitis, and erythema elevatum diutinum, among others. Urticarial vasculitis (UV) is divided into a hypocomplementemic type which frequently has systemic manifestations and a normocomplementemic type which does not.
Yet, while the Dermatologic Addendum adds considerable detail to CHCC2012 and enhances understanding of cutaneous vasculitis, it does not constitute an evidence-based set of diagnostic criteria.

\section{The Diagnostic and Classification Criteria in Vasculitis (DCVAS) Study}

The DCVAS Study is an international effort to develop and validate diagnostic and classification criteria for systemic vasculitis for use in practice and clinical trials (3). Analysis of data from nearly 7,000 patients at 136 sites is underway.

A draft set of diagnostic criteria for AAV has been proposed based on findings from DCVAS, using weighted scores for diagnosis of granulomatosis with polyangiitis (GPA), microscopic polyangiitis (MPA), and eosinophilic granulomatosis with polyangiitis (EGPA) (4). These diagnostic scores achieve high sensitivity and specificity. While they do not rely on ANCA testing to arrive at a diagnosis, ANCA positivity is heavily weighted in the scoring system.

Validated diagnostic criteria are vital for clinical practice and research and may inform understanding of the underlying disease states. Notably, as reflected in the new criteria, AAV is increasingly classified and conceptualized in terms of ANCA type (e.g., PR3, MPO, ANCA-negative) rather than by disease definition or nomenclature (e.g., GPA, MPA, EGPA). The ANCA type offers clinical and prognostic information and facilitates disease differentiation (5-9).

A recent study expands our understanding of cutaneous manifestations of AAV by disease and ANCA type, using data from DCVAS (10). Cutaneous manifestations are common in all AAV subtypes but vary between them, with certain findings more frequently associated with specific diseases. For example, "allergic" manifestations like hives more often occur in EGPA than in other types of AAV $(\mathrm{P}<0.01)$. When present, skin findings are associated with more severe systemic manifestations of vasculitis, such as glomerulonephritis and mononeuritis, with a hazard ratio of $\sim 2.0$ in GPA and EGPA $(\mathrm{P}<0.01)$. Finally, though underutilized, skin biopsy is frequently diagnostic of vasculitis when performed. These findings underscore the diagnostic and prognostic importance of the cutaneous examination in evaluation and management of AAV.

\section{Evidence-based evaluation of cutaneous vasculitis}

Small vessel vasculitis presenting in the skin is frequently 
skin-limited and self-limited. However, important systemic manifestations of vasculitis or underlying disease states may occur, necessitating careful evaluation. Unfortunately, no evidence-based protocol exists to help guide the evaluation of patients presenting with cutaneous vasculitis.

Emerging data from a cohort of patients with biopsyproven small vessel vasculitis of the skin may provide some insights (11). Findings suggest extensive, unguided testing evaluating for systemic involvement is unlikely to be helpful. Tests such as erythrocyte sedimentation rate and C-reactive protein are non-specific and do not aid identification of patients with underlying disease, while ANCA, ANA, screening radiographs (e.g., chest X-ray, computed tomography), cryoglobulins, and other tests can be helpful in certain circumstances but are insensitive and have limited diagnostic value when broadly applied. Tests utilized for screening purposes in this manner, absent other signs or symptoms of systemic disease, have low predictive value and contribute to increased healthcare expenditures. Efforts are underway using these data to create an evidence-based algorithm to help guide the work-up of small vessel vasculitis of the skin using a targeted, stepwise approach $(12,13)$.

\section{Treatment of vasculitis: towards targeted therapies}

Vasculitis subtype, severity, and patient comorbidities drive treatment selection, with a goal of induction and longterm maintenance of disease remission, with minimal drug toxicity. A number of high-quality publications over the last several years have shifted the standard of care for systemic vasculitis toward more targeted, less immunosuppressive regimens. Multicenter collaborative efforts, largely via the Vasculitis Clinical Research Consortium (VCRC), have driven this progress.

\section{Updates in $A A V$}

Nowhere has this shift toward targeted therapies been more pronounced than in the treatment of AAV. Traditional AAV therapies, such as cyclophosphamide and highdose glucocorticoids for induction and azathioprine or methotrexate for maintenance therapy, are life-saving but associated with significant treatment-related side effects and a high relapse rate $(14,15)$. Over the last decade, a paradigm shift toward more targeted, less broadly immunosuppressive therapies has occurred, based on an improved understanding of disease pathogenesis.

\section{Rituximab for $A A V$}

The RAVE and RITUXVASC trials showed that the combination of rituximab plus glucocorticoids is non-inferior to cyclophosphamide plus glucocorticoids for inducing remission in AAV $(16,17)$. In the RAVE trial, within a specified subgroup, patients with relapsing disease who received rituximab did better than those who received cyclophosphamide (16). In addition, loss of PR3 positivity was more frequent in the rituximab treated group (16). In RITUXVASC, all patients treated with rituximab were ANCA negative by 6 months (17). In addition to inducing remission, rituximab has more recently been shown to be an effective therapy for prevention and treatment of relapsed disease $(14,18)$. These trials included patients with GPA and MPA but not EGPA. Rituximab for EGPA is limited to case series and case reports; its use is considered for severe or refractory disease (19).

\section{Plasma exchange in $A A V$}

GPA and MPA with severe kidney disease or pulmonary hemorrhage have traditionally been treated with plasma exchange to reduce circulating ANCAs (20). A recent largescale trial, PEXIVAS, evaluated $>700$ patients with severe GPA and MPA to compare efficacy of plasma exchange $v$ s. no plasma exchange for prevention of death and end-stage kidney disease. The study also evaluated a reduced-dose glucocorticoid regimen (20). The study found that plasma exchange did not result in a lower incidence of end-stage kidney disease or death and that a reduced-dose glucocorticoid regimen was non-inferior to standard regimens (20).

\section{Avacopan: complement patbway inbibition in $A A V$}

Based on evidence that activation of the alternative complement pathway plays a role in the pathogenesis of AAV, studies are underway to evaluate avacopan, a novel C5a receptor inhibitor (21-23). Phase I and II data suggest targeted inhibition of the interaction between $\mathrm{C} 5 \mathrm{a}$ and $\mathrm{C} 5 \mathrm{aR}$ by avacopan can improve outcomes in $\mathrm{AAV}$ and reduce glucocorticoid exposure (24-28). A large, phase III, multicenter, double-blind RCT is now underway (ADVOCATE) (23).

\section{Anti-IL-5 antibodies in EGPA}

Management of EGPA is often suboptimal, with a high risk of relapse and a need for ongoing immunosuppression. 
Clinical observation and genome-wide association studies suggest ANCA status imparts prognostic and genetic meaning. Candidate targets for future therapies have also been identified (29).

IL-5 is a cytokine central to the pathogenesis of eosinophilia in EGPA (30,31). Mepolizumab, a humanized monoclonal antibody to IL-5, demonstrated efficacy for EGPA, with a low incidence of side effects, in open-label studies and a randomized, placebo-controlled trial $(32,33)$. The MIRRA trial in 2017 evaluated mepolizumab at a dose of $300 \mathrm{mg}$ every 4 weeks for relapsing or refractory EGPA (34). Treatment with mepolizumab was associated with more time in remission and a lower relapse rate compared to placebo, allowing for reduced corticosteroid use. Based on these findings, mepolizumab became the first drug approved for EGPA by the Food and Drug Administration (FDA). Nevertheless, only $53 \%$ of participants achieved remission (34).

Benralizumab and reslizumab are other IL-5 antagonists that have been approved for severe asthma (35). Case series suggest improvement in patient-reported outcomes and reduced use of glucocorticoids in those receiving these medications for EGPA (36-38). Clinical trials are now ongoing. (ClinicalTrials.gov Identifiers: NCT04157348 and NCT02947945).

\section{Skin-limited vasculitis}

While large clinical trials have reshaped AAV therapy over the last decade, treatment of skin-limited vasculitis continues to be based on case reports, case series, and expert opinion. There has been only one small, and brief, randomizedcontrolled trial (RCT) of colchicine for treatment of skinlimited small vessel vasculitis (39). However, a multicenter collaborative effort of dermatologists and rheumatologists through the VCRC is currently underway to develop efficacy data for commonly used agents for management of skin-limited vasculitis.

A randomized multicenter trial for isolated skin vasculitis (ARAMIS) is a sequential multiple assignment randomized trial comparing the efficacy of three drugs-azathioprine, colchicine, and dapsone-for treatment of cutaneous small vessel vasculitis, skin-limited immunoglobulin A (IgA) vasculitis (skin-limited Henoch-Schönlein purpura), and cutaneous PAN (40). This study aims to provide evidence regarding the efficacy and comparative effectiveness of these therapies commonly used for management of chronic/ recurring skin-limited vasculitis.

Cutaneous transcriptomics in systemic vasculitis
(CUTIS) is another multicenter collaborative effort of dermatologists and rheumatologists through the VCRC. In CUTIS, cutaneous manifestations of systemic vasculitis are biopsied for detailed histopathologic and transcriptomic analysis. The information obtained through this study has the potential to enhance understanding of disease pathophysiology and may result in targeted approaches to disease management based on the specific inflammatory pathways identified (NCT03004326).

\section{Neutrophil extracellular traps (NETs) and vasculitis}

NETs have been implicated in a range of autoimmune, and inflammatory conditions, including systemic lupus erythematosus, rheumatoid arthritis, and vasculitis (41). NETs are extracellular, web-like structures composed of granule and nuclear constituents such as MPO, PR3, and histones (42). Initially thought to occur with neutrophil death and as a response to invading microbes, recent evidence suggests not all NETosis is associated with cell death (41). A form of vital NETosis, in which neutrophils retain their ability to perform phagocytic functions, has also been described $(41,43)$.

NETs have been shown to occur in AAV in kidney biopsy specimens in the absence of a microbial infection, suggesting ANCAs may perpetuate a cycle of NET production that maintains delivery of antigen-chromatin complexes to the immune system (44). NETs have also been found in skin specimens $(45,46)$, thrombi $(47,48)$, and serum of patients with AAV (49), and in peripheral nerves of patients with MPA-associated neuropathy (50). While an in-depth review of NETosis is beyond the scope of this manuscript, mounting evidence suggests a pathogenic role. Levamisole-associated vasculopathy/vasculitis has been a source of evidence for this pathogenic role of NETosis $(51,52)$. Levamisole acts via muscarinic receptors to stimulate NETosis (53). These NETs contribute to vascular pathology and have been found in the skin of patients with levamisole-induced autoimmunity, forming autoantibodies against NET components, including neutrophil elastase, PR3, MPO, and antinuclear antibodies (54). As additional evidence mounts for NETosis in vasculitis, treatments directed toward inhibiting or reducing the impact of NETs are under investigation (53-55).

\section{Deficiency of adenosine deaminase 2 (DADA2); a monogenic vasculitis}

DADA2 is a monogenic vasculitis which mimics PAN (56-59). 
DADA2 results from loss-of-function mutations in CECR1, the gene that encodes adenosine deaminase 2 (57). Patients often present at a young age with clinical manifestations of systemic vasculitis. These manifestations may include both skin findings (livedo reticularis, nodular vasculitis, digital necrosis, Raynaud disease, and cutaneous ulcers) and systemic findings (lacunar strokes, fever, hypertension, renal and mesenteric aneurysms) (56-61). The spectrum of clinical manifestations is wide-ranging even within the same family, suggesting variability in the penetrance of expression of DADA2 $(56,57,61)$. Dermatologists should be aware that patients with cutaneous manifestations of PAN may have this disease (61). In addition, authors of a recent study suggest that patients who have hepatitis B-negative PAN should be screened for DADA2 (58). In the same study, patients with GPA and MPA were not found to have this genetic mutation. Treatment options are limited; TNF-inhibitors may be considered for severe disease (62). Fresh-frozen plasma has also been utilized in an attempt to restore levels of adenosine deaminase 2, but this has not been shown to be as effective (62). Because the therapeutic approach, treatment, and prognosis differ between DADA2, idiopathic PAN, and other vasculitides, it is important that clinicians are aware of this condition.

\section{Conclusions}

Recent advances have enhanced understanding of vasculitis pathogenesis, evidence-based classification, and targeted treatment approaches, with a shift away from broad immunosuppression toward therapies targeting specific inflammatory pathways. Large-scale, multi-institutional efforts are leading the way to uncover these insights.

While significant progress has been made, more research is needed. Frequently affected and easily accessible, the skin provides an important opportunity for clinical and translational research in vasculitis, speaking to the importance of dermatologic input and expertise as part of collaborative, multidisciplinary networks.

\section{Acknowledgments}

Funding: None.

\section{Footnote}

Provenance and Peer Review: This article was commissioned by the Guest Editors (Drs. Richard D. Sontheimer, M. Kari Connolly, David F. Fiorentino, and Victoria P. Werth) for the series "Rheumatologic Skin Disease" published in Annals of Translational Medicine. The article has undergone external peer review.

Conflicts of Interest: Both authors have completed the ICMJE uniform disclosure form (available at http://dx.doi. org/10.21037/atm-20-6395). The series "Rheumatologic Skin Disease" was commissioned by the editorial office without any funding or sponsorship. The authors have no other conflicts of interest to declare.

Ethical Statement: The authors are accountable for all aspects of the work in ensuring that questions related to the accuracy or integrity of any part of the work are appropriately investigated and resolved

Open Access Statement: This is an Open Access article distributed in accordance with the Creative Commons Attribution-NonCommercial-NoDerivs 4.0 International License (CC BY-NC-ND 4.0), which permits the noncommercial replication and distribution of the article with the strict proviso that no changes or edits are made and the original work is properly cited (including links to both the formal publication through the relevant DOI and the license). See: https://creativecommons.org/licenses/by-nc-nd/4.0/.

\section{References}

1. Jennette JC, Falk RJ, Bacon PA, et al. 2012 revised International Chapel Hill Consensus Conference Nomenclature of Vasculitides. Arthritis Rheum 2013;65:1-11.

2. Sunderkötter CH, Zelger B, Chen KR, et al. Nomenclature of Cutaneous Vasculitis: Dermatologic Addendum to the 2012 Revised International Chapel Hill Consensus Conference Nomenclature of Vasculitides. Arthritis Rheumatol 2018;70:171-84.

3. DCVAS. Accessed August 15, 2020. Available online: https://research.ndorms.ox.ac.uk/public/dcvas/index.php

4. Robson J, Grayson P, Ponte C, et al. Classification Criteria for the ANCA-Associated Vasculitides. Rheumatology (Oxford) 2019;58:kez058.050.

5. Monti S, Craven A, Klersy C, et al. Association between age at disease onset of anti-neutrophil cytoplasmic antibody-associated vasculitis and clinical presentation and short-term outcomes. Rheumatology (Oxford) 
2021;60:617-28.

6. Deshayes S, Martin Silva N, Khoy K, et al. Clinical impact of subgrouping ANCA-associated vasculitis according to antibody specificity beyond the clinicopathological classification. Rheumatology (Oxford) 2019;58:1731-9.

7. Mahr A, Katsahian S, Varet H, et al. French Vasculitis Study Group (FVSG) and the European Vasculitis Society (EUVAS). Revisiting the classification of clinical phenotypes of anti-neutrophil cytoplasmic antibodyassociated vasculitis: a cluster analysis. Ann Rheum Dis 2013;72:1003-10.

8. Berti A, Warner R, Johnson K, et al. Brief Report: Circulating Cytokine Profiles and Antineutrophil Cytoplasmic Antibody Specificity in Patients with Antineutrophil Cytoplasmic Antibody-Associated Vasculitis. Arthritis Rheumatol 2018;70:1114-21.

9. Jennette JC, Falk RJ. Pathogenesis of antineutrophil cytoplasmic autoantibody-mediated disease. Nat Rev Rheumatol 2014;10:463-73.

10. Micheletti RG, Chiesa Fuxench Z, Craven A, et al. Cutaneous Manifestations of Antineutrophil Cytoplasmic Antibody-Associated Vasculitis. Arthritis Rheumatol 2020;72:1741-7.

11. Walls AC, Din RS, Okhovat JP, et al. Retrospective Analysis of Symptomatology, Comorbidities and Diagnostic Evaluation of Adults Presenting with Initial Episode of Leukocytoclastic Vasculitis [abstract]. Medical Dermatology Society Annual Meeting; Washington, DC; March 3, 2016.

12. Micheletti RG, Werth VP. Small vessel vasculitis of the skin. Rheum Dis Clin North Am 2015;41:21-32.

13. Micheletti RG. Cutaneous vasculitis in rheumatologic disease: Current concepts of skin and systemic manifestations. Clin Dermatol 2018;36:561-6.

14. Smith RM, Jones RB, Specks U, et al. Rituximab as therapy to induce remission after relapse in ANCAassociated vasculitis. Ann Rheum Dis 2020;79:1243-9.

15. Frohnert PP, Sheps SG. Long-term follow-up study of periarteritis nodosa. Am J Med 1967;43:8-14.

16. Stone JH, Merkel PA, Spiera R, et al. Rituximab versus cyclophosphamide for ANCA-associated vasculitis. N Engl J Med 2010;363:221-32.

17. Jones RB, Tervaert JW, Hauser T, et al. Rituximab versus cyclophosphamide in ANCA-associated renal vasculitis. $\mathrm{N}$ Engl J Med 2010;363:211-20.

18. Guillevin L, Pagnoux C, Karras A, et al. Rituximab versus azathioprine for maintenance in ANCA-associated vasculitis. N Engl J Med 2014;371:1771-80.
19. Groh M, Pagnoux C, Baldini C, et al. Eosinophilic granulomatosis with polyangiitis (Churg-Strauss) (EGPA) Consensus Task Force recommendations for evaluation and management. Eur J Intern Med 2015;26:545-53.

20. Walsh M, Merkel PA, Peh CA, et al. Plasma Exchange and Glucocorticoids in Severe ANCA-Associated Vasculitis. N Engl J Med 2020;382:622-31.

21. Brilland B, Garnier AS, Chevailler A, et al. Complement alternative pathway in ANCA-associated vasculitis: Two decades from bench to bedside. Autoimmun Rev 2020;19:102424.

22. Schreiber A, Xiao H, Jennette JC, et al. C5a receptor mediates neutrophil activation and ANCA-induced glomerulonephritis. J Am Soc Nephrol 2009;20:289-98.

23. Merkel PA, Jayne DR, Wang C, et al. Evaluation of the Safety and Efficacy of Avacopan, a C5a Receptor Inhibitor, in Patients with Antineutrophil Cytoplasmic AntibodyAssociated Vasculitis Treated Concomitantly with Rituximab or Cyclophosphamide/Azathioprine: Protocol for a Randomized, Double-Blind, Active-Controlled, Phase 3 Trial. JMIR Res Protoc 2020;9:e16664.

24. Bekker P, Dairaghi D, Seitz L, et al. Characterization of Pharmacologic and Pharmacokinetic Properties of CCX168, a Potent and Selective Orally Administered Complement 5a Receptor Inhibitor, Based on Preclinical Evaluation and Randomized Phase 1 Clinical Study. PLoS One 2016;11:e0164646.

25. Jayne DRW, Bruchfeld AN, Harper L, et al. Randomized Trial of C5a Receptor Inhibitor Avacopan in ANCAAssociated Vasculitis. J Am Soc Nephrol 2017;28:2756-67.

26. Merkel PA, Niles J, Jimenez R, et al. A Randomized Clinical Trial of CCX168, an Orally Administered C5aR Inhibitor for Treatment of Patients with ANCA-Associated Vasculitis [abstract]. Arthritis Rheumatol 2016;68(suppl 10). https://acrabstracts.org/abstract/a-randomizedclinical-trial-of-ccx168-an-orally-administered-c5arinhibitor-for-treatment-of-patients-with-anca-associatedvasculitis/. Accessed February 2, 2021.

27. Robson J, Doll H, Suppiah R, et al. Glucocorticoid treatment and damage in the anti-neutrophil cytoplasm antibody-associated vasculitides: long-term data from the European Vasculitis Study Group trials. Rheumatology (Oxford) 2015;54:471-81.

28. Caplan A, Fett N, Rosenbach M, et al. Prevention and management of glucocorticoid-induced side effects: A comprehensive review: A review of glucocorticoid pharmacology and bone health. J Am Acad Dermatol 2017;76:1-9. 
29. Lyons PA, Peters JE, Alberici F, et al. Genome-wide association study of eosinophilic granulomatosis with polyangiitis reveals genomic loci stratified by ANCA status. Nat Commun 2019;10:5120.

30. Jakiela B, Szczeklik W, Plutecka H, et al. Increased production of IL-5 and dominant Th2-type response in airways of Churg-Strauss syndrome patients. Rheumatology (Oxford) 2012;51:1887-93.

31. Hellmich B, Csernok E, Gross WL. Proinflammatory cytokines and autoimmunity in Churg-Strauss syndrome. Ann N Y Acad Sci 2005;1051:121-31.

32. Ennis D, Lee JK, Pagnoux C. Mepolizumab for the treatment of eosinophilic granulomatosis with polyangiitis. Expert Opin Biol Ther 2019;19:617-30.

33. Steinfeld J, Bradford ES, Brown J, et al. Evaluation of clinical benefit from treatment with mepolizumab for patients with eosinophilic granulomatosis with polyangiitis. J Allergy Clin Immunol 2019;143:2170-7.

34. Wechsler ME, Akuthota P, Jayne D, et al. EGPA Mepolizumab Study Team. Mepolizumab or Placebo for Eosinophilic Granulomatosis with Polyangiitis. N Engl J Med 2017;376:1921-32.

35. Nagase H, Ueki S, Fujieda S. The roles of IL-5 and anti-IL-5 treatment in eosinophilic diseases: Asthma, eosinophilic granulomatosis with polyangiitis, and eosinophilic chronic rhinosinusitis. Allergol Int 2020;69:178-86.

36. Padoan R, Chieco Bianchi F, Marchi MR, et al. Benralizumab as a glucocorticoid-sparing treatment option for severe asthma in eosinophilic granulomatosis with polyangiitis. J Allergy Clin Immunol Pract 2020;8:32253227.e2.

37. Coppola A, Flores KR, De Filippis F. Rapid onset of effect of benralizumab on respiratory symptoms in a patient with eosinophilic granulomatosis with polyangiitis. Respir Med Case Rep 2020;30:101050.

38. Kent BD, d'Ancona G, Fernandes M, et al. Oral corticosteroid-sparing effects of reslizumab in the treatment of eosinophilic granulomatosis with polyangiitis. ERJ Open Res 2020;6:00311-2019.

39. Sais G, Vidaller A, Jucglà A, et al. Colchicine in the treatment of cutaneous leukocytoclastic vasculitis. Results of a prospective, randomized controlled trial. Arch Dermatol 1995;131:1399-402.

40. Micheletti RG, Pagnoux C, Tamura RN, et al. Protocol for a randomized multicenter study for isolated skin vasculitis (ARAMIS) comparing the efficacy of three drugs: azathioprine, colchicine, and dapsone. Trials 2020;21:362.
41. Jorch SK, Kubes P. An emerging role for neutrophil extracellular traps in noninfectious disease. Nat Med 2017;23:279-87.

42. Brinkmann V, Reichard U, Goosmann C, et al. Neutrophil extracellular traps kill bacteria. Science 2004;303:1532-5.

43. Yipp BG, Kubes P. NETosis: how vital is it? Blood 2013;122:2784-94.

44. Kessenbrock K, Krumbholz M, Schönermarck U, et al Netting neutrophils in autoimmune small-vessel vasculitis. Nat Med 2009;15:623-5.

45. Abreu-Velez AM, Smith JG Jr, Howard MS. Presence of neutrophil extracellular traps and antineutrophil cytoplasmic antibodies associated with vasculitides. N Am J Med Sci 2009;1:309-13.

46. Sangaletti S, Tripodo C, Chiodoni C, et al. Neutrophil extracellular traps mediate transfer of cytoplasmic neutrophil antigens to myeloid dendritic cells toward ANCA induction and associated autoimmunity. Blood 2012;120:3007-18.

47. Imamoto T, Nakazawa D, Shida H, et al. Possible linkage between microscopic polyangiitis and thrombosis via neutrophil extracellular traps. Clin Exp Rheumatol 2014;32:149-50.

48. Nakazawa D, Tomaru U, Yamamoto C, et al. Abundant neutrophil extracellular traps in thrombus of patient with microscopic polyangiitis. Front Immunol 2012;3:333.

49. Söderberg D, Kurz T, Motamedi A, et al. Increased levels of neutrophil extracellular trap remnants in the circulation of patients with small vessel vasculitis, but an inverse correlation to anti-neutrophil cytoplasmic antibodies during remission. Rheumatology (Oxford) 2015;54:2085-94.

50. Takeuchi H, Kawasaki T, Shigematsu K, et al. Neutrophil extracellular traps in neuropathy with anti-neutrophil cytoplasmic autoantibody-associated microscopic polyangiitis. Clin Rheumatol 2017;36:913-7.

51. Lood C, Hughes GC. Neutrophil extracellular traps as a potential source of autoantigen in cocaine-associated autoimmunity. Rheumatology (Oxford) 2017;56:638-43.

52. Carmona-Rivera C, Purmalek MM, Moore E, et al. A role for muscarinic receptors in neutrophil extracellular trap formation and levamisole-induced autoimmunity. JCI Insight 2017;2:e89780.

53. Lee KH, Kronbichler A, Park DD, et al. Neutrophil extracellular traps (NETs) in autoimmune diseases: A comprehensive review. Autoimmun Rev 2017;16:1160-73.

54. Söderberg D, Segelmark M. Neutrophil extracellular traps in vasculitis, friend or foe? Curr Opin Rheumatol 
2018;30:16-23.

55. Frangou E, Vassilopoulos D, Boletis J, et al. An emerging role of neutrophils and NETosis in chronic inflammation and fibrosis in systemic lupus erythematosus (SLE) and ANCA-associated vasculitides (AAV): Implications for the pathogenesis and treatment. Autoimmun Rev 2019;18:751-60.

56. Liebowitz J, Hellmann DB, Schnappauf O. Thirty Years of Followup in 3 Patients with Familial Polyarteritis Nodosa due to Adenosine Deaminase 2 Deficiency. J Rheumatol 2019;46:1059-60.

57. Navon Elkan P, Pierce SB, Segel R, et al. Mutant adenosine deaminase 2 in a polyarteritis nodosa vasculopathy. N Engl J Med 2014;370:921-31.

58. Schnappauf O, Sampaio Moura N, Aksentijevich I, et al. Sequence-Based Screening of Patients With Idiopathic Polyarteritis Nodosa, Granulomatosis With Polyangiitis, and Microscopic Polyangiitis for Deleterious Genetic

Cite this article as: Caplan A, Micheletti RG. Advances in cutaneous vasculitis research and clinical care. Ann Transl Med 2021;9(5):439. doi: 10.21037/atm-20-6395
Variants in ADA2. Arthritis Rheumatol 2021;73:512-9.

59. Zhou Q, Yang D, Ombrello AK, et al. Early-onset stroke and vasculopathy associated with mutations in ADA2. N Engl J Med 2014;370:911-20.

60. Ganhão S, Loureiro GB, Oliveira DR, et al. Two cases of ADA2 deficiency presenting as childhood polyarteritis nodosa: novel ADA2 variant, atypical CNS manifestations, and literature review. Clin Rheumatol 2020;39:3853-60.

61. Gonzalez Santiago TM, Zavialov A, Saarela J, et al. Dermatologic Features of ADA2 Deficiency in Cutaneous Polyarteritis Nodosa. JAMA Dermatol 2015;151:1230-4. Erratum in: JAMA Dermatol. 2016 Sep 1;152(9):1065. doi: 10.1001/jamadermatol.2016.2640. JAMA Dermatol. 2016 Sep 1;152(9):1065. doi: 10.1001/jamadermatol.2016.2595.

62. Ombrello AK, Qin J, Hoffmann PM, et al. Treatment Strategies for Deficiency of Adenosine Deaminase 2. N Engl J Med 2019;380:1582-4. 